\title{
Beyond expectation: a case for nonpersonal contextual factors in a more comprehensive approach to the placebo effect and the contribution of environmental psychology
}

\author{
This article was published in the following Dove Press journal: \\ Psychology Research and Behavior Management \\ 30 October 2015 \\ Number of times this article has been viewed
}

\author{
Stefan Sütterlin ${ }^{1,2}$ \\ Lars E Egner' \\ Ricardo G Lugo' \\ Slawomir Wojniusz ${ }^{2,3}$ \\ 'Section of Psychology, Lillehammer \\ University College, Lillehammer, \\ ${ }^{2}$ Department of Psychosomatic \\ Medicine, Division of Surgery \\ and Clinical Neuroscience, Oslo \\ University Hospital - Rikshospitalet, \\ Oslo, ${ }^{3}$ Department of Physiotherapy, \\ Faculty of Health Sciences, Oslo \\ and Akershus University College of \\ Applied Sciences, Oslo, Norway
}

\begin{abstract}
Creating an optimized health care environment to maximize the probability and magnitude of placebo effects draws on a number of well-researched mechanisms such as the patient's positive expectation toward treatment outcome. Patient-centered communication styles influence expectations and can thus be considered as a form of supplemental treatment. Unconsciously processed contextual triggering and facilitating placebo effects are omnipresent in clinical settings as well as in all other social and physical environments. Contextual cues in both the social and physical domain exert influences on the recipient's emotional state and recreational experiences. While the majority of research focuses on improving the patients expectations, classical conditioning effects of nonsocial contextual factors have been largely neglected in discussions on practical implementation of placebo-enhancing environments. Built on the empirically well-supported argument that conditioning processes act as a powerful tool to mobilize self-healing resources just as verbally induced expectations do, we argue for a stronger consideration of the effects of permanent, nonsocial and nonverbal environmental contexts. Environmental psychology is a new field of research within the psychological domain and offers a toolbox of opportunities for medical psychological research and health care practitioners to improve the treatment outcomes and benefits of health care environments.
\end{abstract}

Keywords: placebo, expectation, conditioning, environmental psychology

\section{Introduction}

In their article "How can placebo effects best be applied in clinical practice? A narrative review", Bystad et $\mathrm{al}^{1}$ make a case for the potential benefits of additive use of placebo effects in health care. The authors discuss how health care professionals can make use of placebo effects and conclude that these can be maximized by influencing the patients' expectation via personal communication with the purpose of building up trust and respect. Placebo effects have a significant impact on treatment success and personal interaction with health care professionals can contribute to the understanding of placebo as a form of supplemental treatment. Bystad et al ${ }^{1}$ define placebo effects as "psychological and/or physiological responses that follow the administration of active and non-active substances when coupled with affirmation of the treatment effects". ${ }^{1}$ In this commentary, we will apply a comprehensive definition of placebo effects as it is commonly used in pharmaceutical studies. Following this approach, placebo effects can be triggered by contextual stimuli other than exclusively "medication-like" substances. Placebo effects are triggered by contextual factors such as the appearance of persons who administer medical treatment,
Correspondence: Stefan Sütterlin Section of Psychology,

Lillehammer University College,

Gudbrandsdalsvegen 350,

2624 Lillehammer, Lillehammer, Norway

Tel +479807 6685

Email stefan.sutterlin@hil.no 
the amount of time and material resources invested in the treatment and physical environment. ${ }^{2}$ In our commentary, we aim to inspire future research to investigate contextual factors also in terms of nonpersonal (ie, physical) environments.

Positive health benefits caused by these contextual stimuli that are not inherent in or intended by the medical treatment are considered placebo effects ${ }^{2}$ in the field of medicine, but are the actual subject of investigation and medium of intended effects in environmental psychology. This differentiation underlines the relativity and context-dependent meaning of the term placebo: An unspecific "side"-effect of a medication can constitute a specific effect from a psychological perspective. In the current paper, we will refer to the term placebo as a factor adding to specific pharmacological effects and consider psychological influences on well-being as contextual stimuli.

Bystad et $\mathrm{al}^{1}$ review the literature on the factors influencing probability and magnitude of placebo effects and conclude with specific recommendations to be implemented in clinical practice. The reviewed literature provides an accurate picture of the present-day research on placebo in clinical practice. Typically, the investigation of factors deemed suitable for powerful placebo effects concentrates on a patient's positive expectations toward the treatment outcome ("... doctors may best benefit from placebo effects by influencing the patient's expectation"). ${ }^{1}$ To promote placebo usage in clinical practice, the authors particularly emphasize patient-centered communication style (ie, "developing trust and respect, exploring patient's values, speaking positively about treatments and providing reassurance and encouragement"). ${ }^{1}$ While the authors make a convincing point that communication style is the primary facilitator of placebo effect, the awareness and utilization of nonpersonal, placebo promoting factors can further increase its supplemental therapeutic effect. In this commentary, we argue that placebo effects go beyond patient-clinician communication and also include nonpersonal contextual factors such as the physical environment.

Nonpersonal contextual factors are defined as consciously or unconsciously perceived stimuli in a clinical setting beyond persons and social interaction. In the following, we aim to discuss the role of nonpersonal contextual factors in relation to classical conditioning and emotion regulation and suggest ways in which environmental psychology can contribute to additional placebo effects in clinical settings.

\section{Placebo effects evoked by classical conditioning}

The relevance of positive patient expectations evoked or at least influenced by doctor-patient communication styles for the placebo effect are undisputed, have been replicated in numerous studies, and found their way into textbooks and curricula of medical psychology. ${ }^{3}$ As Bystad et al ${ }^{1}$ point out, personal communication is a crucial part of health promotion in health care environments. In our opinion, stressing the personal factor and creating an empathic environment is the most important single factor (for a theoretical model on empathy in medicine see Decety and Fotopoulou). ${ }^{4}$ Nonverbal, nonpersonal contextual influences, however, might also contribute to beneficial health effects and thus increase the benefits of placebo enhancement in clinical settings. A large body of research suggests that placebo effects explained with conditioning effects showed comparable robustness and magnitude as placebo effects triggered by expectation-inducing verbal instructions. ${ }^{5}$ According to Rutherford and Roose, "placebo responses represent a type of non-conscious learning in which an individual associates improvement in symptoms (unconditioned response) with neutral stimuli such as pills, health care providers, or procedures (conditioned stimulus) that themselves become capable of eliciting an effect (conditioned response)". ${ }^{6}$ Building upon this definition, placebo responses (ie, effects not directly intended and intentionally addressed in a medical treatment) might also be triggered by the physical, nonpersonal environment. As an example, objects brought from home, pictures of familiar natural environments, photos, or a generally cozy architectural environment might trigger conditioned responses promoting recovery processes. ${ }^{7,8}$

Thus, conditioning processes act as another powerful tool to mobilize self-healing resources, just as verbally induced expectations do. Comparative research on endogenous analgesia suggests that patient expectations and classical conditioning effects contribute independently to placebo effects. ${ }^{5}$ When applied in concert, however, additional interaction effects occur that exceed the additive value of both mechanisms per se. ${ }^{9}$ We consider the influence of conditioning to be an often underestimated and not yet sufficiently appreciated resource for improvement in treatment outcomes. Conditioning does not affect expectations or even requires conscious processing. Instead, a large variety of contextual stimuli, including permanently present nonpersonal situational or structural stimuli can potentially trigger the mobilization of a patient's emotion regulatory resources.

\section{Contributions by environmental psychology}

Environmental psychology studies the ways in which people perceive and respond to the physical environment 
and offers tools to mobilize emotion regulation resources using nonverbal, nonpersonal contextual stimuli beyond consciousness and expectation. ${ }^{10-12} \mathrm{An}$ increasing amount of research is investigating how and why interventions such as manipulating the window view, noise and plant density affects patient recovery as well as work environments. ${ }^{13}$ The most frequently studied variable is noise, which has been shown to affect sleep, increase stress, and affect emotional states. Hagerman et $\mathrm{al}^{14}$ assigned coronary artery disease patients rooms with sound-reflecting ceiling tiles (bad acoustics) and sound-absorbing tiles of similar appearance (good acoustics). Patients in the good acoustics setting had lower pulse amplitude during the night in intensive coronary care, a lower rate of rehospitalization at 1- and 3-month follow-up and considered staff attitude to be much better. Further intervention to minimize noise include lowering telephone volumes, placing occupants in single rooms, and dimming lights to lower voices. ${ }^{7}$ Reducing noise is probably the most straightforward way of improving both patient and staff stay.

View is probably the most psychologically interesting health care environment factor. Perhaps the best known study concerning the restorative effects of nature concluded that patients who had a window view to nature had shorter stays at the hospital, fewer negative evaluative comments and took less potent analgesic medication. ${ }^{8}$ In general, the representation of nature, either through a window view or art, has been shown to be associated with health benefits. A window view might be preferred because it also shows the time of day through daylight, assisting circadian rhythms, and with modern technology, this feature can be achieved through virtual windows. While constructing windows to view nature is the most effective intervention, it is most certainly also the most expensive. Making rooms more visually appealing and introducing artworks, preferably of nature, can be a cost-effective alternative. It is important to focus on the practical aspects of improving a scene, as the subjective perception of a scene affects the patient. Studies have shown that cortisol levels were lower for patients staying at hospital rooms redecorated with paneling found at homes, ${ }^{15}$ suggesting that rooms that not only have a better view and are less noisy give a better patient experience, but the subjective feel of the room improves the patient's time in the health setting.

Making a patient feel comfortable and at home reduces stress and might improve the patient's recovery. ${ }^{16}$ The optimal clinical setting maximizes patient comfort and well-being while maintaining medical functionality. This includes several components that have been shown to reduce stress, such as a low window/natural art to wall area ratio, a minimal level of noise, and making sure that patient areas closely resemble more "homey" environments. Obviously, creating a benevolent nonsocial environment is bound to location, it thus remains unclear in which way this knowledge can contribute to outpatient treatments. The fact that physical stimuli are bound to location underlines the relevance of less location-bound social factors as described by Bystad et al, with nonpersonal factors as useful complement.

\section{Conclusion}

Creating an optimized environment that maximizes probability and magnitude of placebo effects draws on a number of well-researched mechanisms. A central mechanism is the patient's positive expectation toward treatment outcome. Positive expectations are likely to mobilize emotion regulation resources related to the activation of regions that may be "part of a general circuit underlying the voluntary regulation of affective responses". ${ }^{5}$ These emotion regulatory mechanisms are not solely triggered by conscious expectations following verbal communication. Unconsciously processed situational cues are omnipresent in every clinical situation as well as in all other environments and exert influences on the recipient's emotional state and recreational experiences. Classical conditioning and conscious processing of environmental stimuli are nonpersonal contextual factors that are also the mechanisms via which patients mobilize emotional regulatory resources, elicit positive expectations, and thus promote positive treatment outcomes that add to the actual active substance and effects of verbal communication. Whether the exposure to environmental stimuli, such as nature, is related to earlier learning experiences, such as classical conditioning, should be addressed in future research. Environmental psychology offers a toolbox of opportunities to maximize the health benefits of health care environments and can thus add on to the effects described by Bystad et al. ${ }^{1}$

\section{Disclosure}

The authors report no conflicts of interest in this work.

\section{References}

1. Bystad M, Bystad C, Wynn R. How can placebo effects best be applied in clinical practice? A narrative review. Psychol Res Behav Manag. 2015;8:41-45.

2. Brown WA. The Placebo Effect in Clinical Practice. New York: Oxford University Press; 2013.

3. Benedetti F. Placebo effects: understanding the mechanisms in health and disease. Z Med Psychol. 2010;19:180-180.

4. Decety J, Fotopoulou A. Why empathy has a beneficial impact on others in medicine: unifying theories. Front Behav Neurosci. 2015;8:457. 
5. Benedetti F, Mayberg HS, Wager TD, Stohler CS, Zubieta JK. Neurobiological mechanisms of the placebo effect. J Neurosci. 2005; 25(45):10390-10402.

6. Rutherford BR, Roose SP. A model of placebo response in antidepressant clinical trials. Am J Psychiatry. 2013;170(7):723-733.

7. Devlin AS, Arneill AB. Health care environments and patient outcomes a review of the literature. Environ Behav. 2003;35(5):665-694.

8. Ulrich RS. View through a window may influence recovery from surgery. Science. 1984;224(4647):420-421.

9. Hoffman GA, Harrington A, Fields HL. Pain and the placebo: what we have learned. Perspect Biol Med. 2005;48(2):248-265.

10. Cropley M, Flaxman P, Gatersleben B. Recovery and restoration: the interface between the individual and the environment. Paper presented at: International Workshop at Lillehammer University College (HiL); June 10-12, 2013; Hamar, Norway.

11. Johnsen SÅK. Exploring the use of nature for emotion regulation: associations with personality, perceived stress, and restorative outcomes. Nordic Psychol. 2013;65(4):306-321.
12. Johnsen SÅK, Rydstedt LW. Active Use of the Natural Environment for Emotion Regulation. Europe's Journal of Psychology. 2013;9(4): 798-819.

13. Nieuwenhuis M, Knight C, Postmes T, Haslam SA. The relative benefits of green versus lean office space: three field experiments. J Exp Psychol Appl. 2014;20(3):199.

14. Hagerman I, Rasmanis G, Blomkvist V, Ulrich R, Eriksen CA, Theorell T. Influence of intensive coronary care acoustics on the quality of care and physiological state of patients. Int J Cardiol. 2005; 98(2):267-270.

15. Ohta H, Maruyama M, Tanabe Y, et al. Effects of redecoration of a hospital isolation room with natural materials on stress levels of denizens in cold season. Int J Biometeorol. 2008;52(5):331-340.

16. Fornara F, Andrade CC. Health care environments. In: Clayton SD, editor. The Oxford Handbook of Environmental and Conservation Psychology. New York: Oxford University Press; 2012:295-315.

\section{Publish your work in this journal}

Psychology Research and Behavior Management is an international, peerreviewed, open access journal focusing on the science of psychology and its application in behavior management to develop improved outcomes in the clinical, educational, sports and business arenas. Specific topics covered include: Neuroscience, memory \& decision making; Behavior

\section{Dovepress}

modification \& management; Clinical applications; Business \& sports performance management; Social and developmental studies; Animal studies. The manuscript management system is completely online and includes a quick and fair peer-review system. Visit http://www.dovepress. com/testimonials.php to read real quotes from published authors. 